\title{
An Updated Multi-Wavelength Radio and Optical Catalog of Quasars and Radio Galaxies
}

\author{
Amy E. Kimball ${ }^{1}$ and Željko Ivezić ${ }^{2}$ \\ ${ }^{1}$ CSIRO Astronomy and Space Science, \\ Australia Telescope National Facility, \\ PO Box 76, Epping NSW, 1710, Australia \\ email: Amy.Kimball@csiro.au \\ ${ }^{2}$ Department of Astronomy, University of Washington, \\ Box 351580, Seattle WA 98195-1580, USA \\ email: ivezic@astro.washington.edu
}

\begin{abstract}
We present a catalog of millions of radio sources, created by consolidating large-area radio and optical surveys GB6 $(6 \mathrm{~cm})$, FIRST $(20 \mathrm{~cm})$, NVSS $(20 \mathrm{~cm})$, WENSS $(92 \mathrm{~cm})$, VLSS $(4 \mathrm{~m})$, and SDSS DR9 (optical). The region where all surveys overlap covers $3269 \mathrm{deg}^{2}$ in the North Galactic Cap, and contains >160,000 20-cm sources, with about 12,000 detected in all five radio surveys and over one-third detected optically. Combining parameters from the sky surveys allows easy and efficient classification by radio and optical morphology and radio spectral index. The catalog is available at http://www.atnf.csiro.au/people/Amy.Kimball/radiocat.shtml.
\end{abstract}

Keywords. catalogs, radio continuum: general, quasars: general, galaxies: general

We present an updated version of the unified radio catalog published by Kimball \& Ivezić (2008). That catalog comprised sources detected at $20 \mathrm{~cm}$ by the Faint Images of the Radio Sky at Twenty cm survey (FIRST; Becker, White, \& Helfand 1995) and/or the NVSS (Condon et al. 2008), with supplemental data (when available) from the Green Bank 6-cm survey (Gregory et al. 1996), at $92 \mathrm{~cm}$ from the Westerbork Northern Sky Survey (Rengelink et al. 1997) and in the optical by the Sloan Digital Sky Survey (SDSS).

The main addition to the catalog is the inclusion of $4-\mathrm{m}(74-\mathrm{GHz})$ data detections (when available) from the VLA Low-Frequency Sky Survey Redux† (VLSS; Lane et al. 2012). The VLSS covers the majority of the sky north of $\delta=-40^{\circ}$ (i.e., the majority of the sky area covered by the original unified radio catalog), with a sensitivity limit of about $700 \mathrm{mJy}$, significantly brighter than the other radio surveys included in the catalog. As a low-frequency survey, it is more sensitive to steep-spectrum sources $(\alpha<-0.5$ where $\left.f_{\nu} \propto \nu^{\alpha}\right)$ than to flat-spectrum sources $(\alpha \sim 0)$. Approximately $10 \%$ of the entries in the updated catalog have a VLSS counterpart. We find an optimal matching radius of $\sim 65^{\prime \prime}$ between VLSS and FIRST/NVSS.

The updated catalog also includes updated data from FIRST (updated 24 Feb 2012), as well as the latest version of NVSS (version 41). The new data reductions from those surveys have resulted in modified source lists (especially at low signal-to-noise) and small variations in measured parameters; however, the overall properties of the radio source populations in the unified catalog have not changed significantly.

Additionally, this version of the catalog incorporates optical data from the Ninth Data Release (DR9) of the SDSS (Ahn et al. 2012); the previous version of the catalog used

$\dagger$ http://www.cv.nrao.edu/vlss/VLSSlist.shtml 


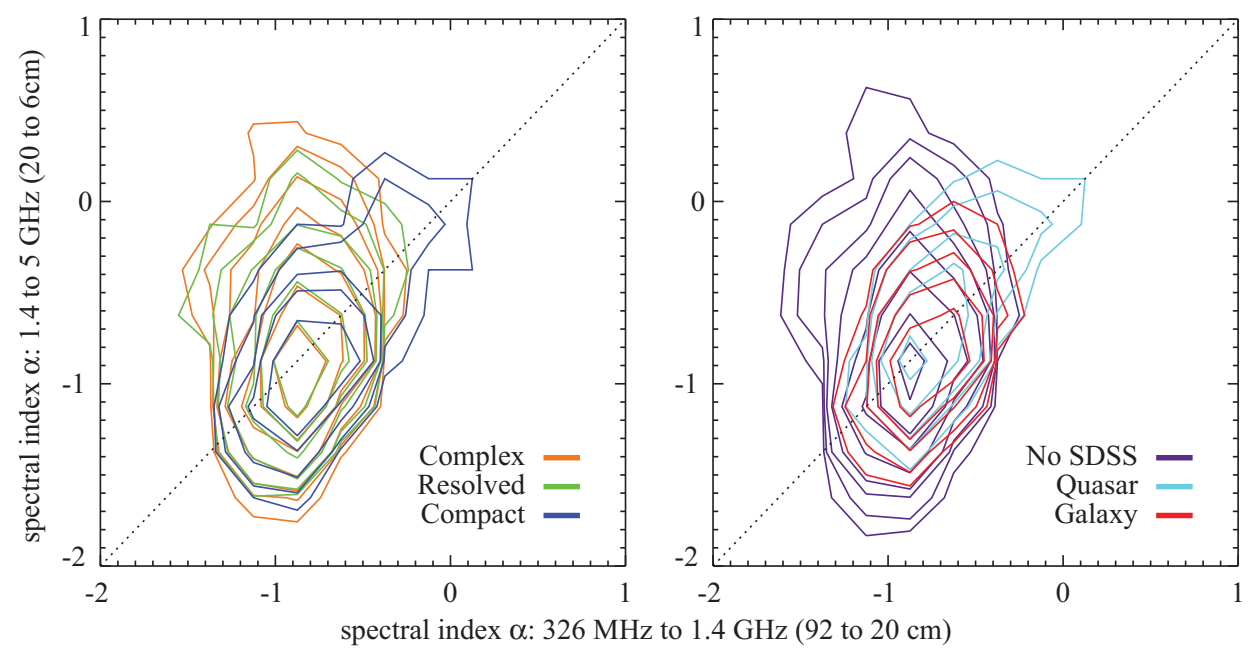

Figure 1. Spectral index distributions for the 12,000 sources detected in all five radio surveys; $\alpha<0$ implies flux density $f_{\nu}$ rising with increased wavelength. The dotted line indicates sources with constant spectral index. Left: comparison by radio morphology class. Spectral indices of compact sources are typically constant from $92 \mathrm{~cm}$ to $6 \mathrm{~cm}$, while many resolved and complex sources have spectra that flatten out toward shorter wavelengths. Right: comparison by optical SDSS identification. Quasars tend to have flatter spectral indices than radio galaxies. (Color figure is available in the electronic version of these Proceedings.)

the Sixth Data Release (DR6) of the SDSS (Adelman-McCarthy et al. 2008). As a result, the number of sources in the radio catalog that have optical spectra has increased by almost $60 \%$.

Figure 1 illustrates morphological and spectral index characteristics of the population of sources detected in all five radio surveys $\left(12,000\right.$ in the $3269 \mathrm{deg}^{2}$ where the surveys overlap). The left panel shows spectral index distributions of three radio morphology classes defined at $20 \mathrm{~cm}$ (see Kimball \& Ivezić 2008): compact (unresolved), resolved, and complex (extended). The right panel shows spectral index distributions of three optical morphology classes: galaxy (resolved), quasar (unresolved), and optically undetected. Quasars tend to have flatter spectral indices (suggesting flat-spectrum radio-jet core sources) than galaxies (suggesting steep-spectrum radio lobes). Similarly, the compact class has more flat-spectrum sources than the resolved or complex classes. Spectral indices of compact sources are more likely to remain constant from $92 \mathrm{~cm}$ to $6 \mathrm{~cm}$, while many resolved and complex sources have spectra that flatten out toward shorter wavelengths.

\section{References}

Adelman-McCarthy, J. K. et al. 2008 ApJS, 175-297

Ahn, C. P., et al. 2012 ApJS, 203, 21

Becker, R. H., White, R. L., \& Helfand, D. J. 1995 ApJ, 450, 559

Lane, W. M., Cotton, W. D., Helmboldt, J., \& Kassim, N. E. 2012 Radio Sci., v. 47, RS0K04

Condon, J. J., et al. 1998 AJ, 115, 1693

Gregory P. C., Scott, W. K., Douglas, K., \& Condon, J. J. 1996 ApJS, 103, 427

Kimball, A. E. \& Ivezić, Ž. 2008, $A J, 136,684$

Rengelink, R. B., et al. 1997 A\&AS, 124, 259 